Artigo Original

\title{
Avaliação dos Efeitos da Transição de Múltiplas Injeções Diárias de Insulina para Infusão Subcutânea Contínua de Insulina no Tratamento Intensivo da Diabetes Mellitus Tipo 1
}

\author{
Sofia Castro Oliveira ${ }^{\mathrm{a}, \mathrm{b}, \mathrm{c}^{*}}$, Celestino Neves ${ }^{\mathrm{a}, \mathrm{b}, \mathrm{c}}$, César Esteves ${ }^{\mathrm{a}}$, João Sérgio Neves ${ }^{\mathrm{ab}, \mathrm{c}}$, Ana Isabel Oliveira ${ }^{\mathrm{a}}$, Miguel \\ Pereira $^{\mathrm{a}}$, Cristina Arteiro ${ }^{\mathrm{a}}$, Anabela Costa ${ }^{\mathrm{a}}$, Maria do Carmo Redondo ${ }^{\mathrm{a}}$, Rui Baltazar ${ }^{\mathrm{a}}$, Davide Carvalho ${ }^{\mathrm{a}, \mathrm{b}, \mathrm{c}}$
}

\author{
${ }^{a}$ Serviço de Endocrinologia, Diabetes e Metabolismo do Centro Hospitalar Universitário de São João, Porto, Portugal \\ ${ }^{b}$ Faculdade de Medicina da Universidade do Porto, Porto, Portugal \\ ' Instituto de Investigação e Inovação em Ciências da Saúde (I3S), Universidade do Porto, Porto, Portugal
}

\section{INFORMAÇÃO SOBRE O ARTIGO}

Historial do artigo:

Received/ Recebido: 2019-01-22

Accepted/Aceite: 2019-04-08

Online: 2019-09-30

Palavras-chave:

Automonitorização da Glicemia

Diabetes Mellitus Tipo 1/tratamento

Injeções Subcutâneas

Insulina/administração e dosagem

Sistemas de Infusão de Insulina

\begin{abstract}
R E S U M O
Introdução: A insulinoterapia intensiva constitui, atualmente, o tratamento de eleição na diabetes mellitus (DM) tipo 1, a qual inclui as múltiplas injeções diárias de insulina (MIDI) e a infusão subcutânea contínua de insulina (ISCI). Esta última, tem vindo a tornar-se o modo terapêutico preferencial, visto mimetizar melhor a ação pancreática fisiológica, sendo, contudo, limitada a evidência que suporta a sua superioridade relativamente à MIDI. Neste contexto, o objetivo do nosso estudo foi avaliar os efeitos da transição de MIDI para ISCI no tratamento intensivo da DM tipo 1.

Material e Métodos: Estudo longitudinal retrospetivo em doentes previamente sob MIDI que transitaram para ISCI entre 2006 e 2014. Foram recolhidos dados referentes ao peso, HbA1c, glicemia plasmática, perfil lipídico, creatinina, frequência semanal de episódios de hipoglicemia e de hiperglicemia e presença de complicações microvasculares. Os efeitos da transição para ISCI foram também comparados de acordo com os seguintes subgrupos: HbAlc pré-ISCI $(\leq 7,0 \%$ vs $>7,0 \%)$; idade ( $\leq 35$ anos $v s>35$ anos); sexo (masculino versus feminino); IMC ( $\leq 25 v s>25 \mathrm{~kg} / \mathrm{m} 2)$; duração da doença ( $\leq 15$ anos $v s>15$ anos); dose diária total (DDT) de insulina ( $\leq 45 \mathrm{e}>45$ unidades de insulina); FSI ( $\leq 40$ e $>40)$ e complicações microvasculares (presença versus ausência).

Resultados: A amostra incluiu 85 doentes, com média de idades de $38 \pm 11$ anos, $50(58,8 \%)$ do sexo feminino, com duração média de doença de $21 \pm 9$ anos. Verificou-se uma redução significativa na frequência de eventos de hipo e hiperglicemia após transição para ISCI $(3,0 \pm 5,0$ vs $2,0 \pm 2,2$ por semana, $p=0,001$; e 5,5 $\pm 6,1$ vs 2,5 $\pm 2,6$ por semana, $p=0,05$, respetivamente). Observou-se ainda um maior benefício glicémico no subgrupo de doentes com pior controlo metabólico (HbAlc $>7 \%$ ) relativamente ao grupo de doentes com $\mathrm{HbAlc} \leq 7 \%$ ( $\Delta$ $\mathrm{HbAlc}=-0,55 \%$ vs $0,20 \%$, respetivamente, $p<0,05)$, para os 6 primeiros meses após ISCI, constatando-se, adicionalmente, um aumento significativo nos níveis de HDL-C $(2,81 \pm 10,34 \mathrm{mg} / \mathrm{dL}, p=0,039)$.

Conclusão: Neste estudo, a terapêutica com ISCI demonstrou ser mais eficaz comparativamente à MIDI nos doentes com pior controlo metabólico, verificando-se ainda uma redução significativa da frequência semanal de eventos de hipo e hiperglicemia. Não obstante os resultados animadores associados à ISCI, no futuro, torna-se imperativo a realização de estudos longitudinais mais alargados para avaliar a real eficácia relativa da ISCI no tratamento da DM tipo 1.
\end{abstract}

\section{Assessing the Effects of the Transition from Multiple Daily Insulin Injections (MDI) to Continuous Subcutaneous Insulin Infusion in the Intensive Treatment of Type 1 Diabetes Mellitus}

\section{A B S T R A C T}

Introduction: Intensive insulin therapy is currently the main treatment in type 1 diabetes mellitus (DM) which includes multiple daily insulin injections (MDI) and continuous subcutaneous insulin infusion

\footnotetext{
*Autor Correspondente / Corresponding Author.

E-Mail: sofiacastro.oliveira@gmail.com (Sofia Castro Oliveira)

Serviço de Endocrinologia do Centro Hospitalar Universitário de São João,

Alameda Prof.Hernâni Monteiro

4200-319, Porto
} 
(CSII). The latter has become the preferred therapeutic mode, since it better mimics the physiological pancreatic action, althought there is limited evidence that supports its superiority to MDI. The aim of our study was to assess the effects of the transition from MDI to CSII in the intensive treatment of type $1 \mathrm{DM}$. Material and Methods: A retrospective longitudinal study was performed in MDI patients that transited to CSII between 2006 and 2014. Data were collected regarding to weight, HbA1c, plasma glucose, lipid profile, creatinine, weekly frequency of episodes of hypoglycemia and hyperglycemia and presence of microvascular complications. The effects of the transition to CSII were also compared according to the following subgroups: pre-CSII HbA1c $(\leq 7.0 \% v s>7.0 \%)$; age ( $\leq 35$ years $v s$ $>35$ years); gender (male versus female); BMI $(\leq 25 v s>25 \mathrm{~kg} / \mathrm{m} 2)$; duration of illness ( $\leq 15$ years $v s>15$ years); total daily dose (TDD) of insulin ( $\leq 45$ and $>45$ units of insulin); ISF ( $\leq 40$ and $>40$ ) and microvascular complications (presence versus absence).

Results: The sample included 85 patients, mean age $38 \pm 11$ years, 50 (58.8\%) female, with duration of the disease $21 \pm 9$ years. There was a significant reduction in the frequency of hypo and hyperglycemia events after transition to CSII $(3.0 \pm 5.0$ vs $2 \pm 2.2$ per week, $p=0.001$ and $5.5 \pm 6.1$ vs $2.5 \pm$ 2.6 per week, $p=0.05$, respectively). We also observed a greater glycemic benefit in the subgroups of patients with poorer metabolic control $(\mathrm{HbAlc}>7 \%)$ compared to those with $\mathrm{HbAlc} \leq 7 \%$ ( $\Delta$ $\mathrm{HbA} 1 \mathrm{c}=-0.55 \%$ vs $0.20 \%$, respectively, $p<0.05$ ), for the first 6 months after CSII, being additionally reported a significant increase in HDL-C levels $(2.81 \pm 10.34 \mathrm{mg} / \mathrm{dL}, p=0.039)$.

Conclusion: In this study, CSII therapy was shown to be more effective compared to MDI in patients with poorer metabolic control, being also noted a significant reduction of weekly frequency of hypo and hyperglycemia events. Notwithstanding the encouraging results linked with CSII, in the future, longer longitudinal studies will be mandatory in order to assess the real relative effectiveness of CSII in the treatment of type $1 \mathrm{DM}$.

Introdução

A diabetes mellitus (DM) tipo 1 é uma doença autoimune que se caracteriza pela destruição das células- $\beta$ pancreáticas, resultando na incapacidade de produção endógena de insulina. ${ }^{1}$ Estima-se que esta doença atinja entre 19 a 38 milhões de indivíduos a nível mundial, sendo que, na Europa, este número tem vindo a aumentar a um ritmo de $2 \%-5 \%$ por ano. ${ }^{2}$

$\mathrm{O}$ inadequado controlo glicémico nestes doentes conduz ao desenvolvimento de complicações crónicas e aumento da morbi-mortalidade. ${ }^{1,3-7}$ Deste modo, a otimização do controlo glicémico torna-se fundamental na prevenção das complicações micro e macrovasculares associadas a esta doença, possibilitando uma considerável melhoria na qualidade de vida. ${ }^{1,3-7}$ A crescente necessidade de terapêuticas mais intensivas levou ao surgimento de novas abordagens, como as múltiplas injeções diárias de insulina (MIDI) e, baseadas no recente progresso científico e tecnológico, a infusão subcutânea contínua de insulina (ISCI) e desenvolvimento do pâncreas artificial. ${ }^{8-11}$

Efetivamente, o regime de MIDI e a terapêutica de ISCI representam hoje as duas estratégias de eleição no tratamento intensivo da DM tipo 1.

De acordo com a Direção Geral de Saúde (DGS), consideram-se elegíveis para terapêutica com ISCI os doentes com a devida motivação, auto-monitorização regular da glicemia capilar e capacidade adequada para a sua utilização. Adicionalmente, deverão ainda obedecer aos seguintes critérios (Tabela 1): controlo meta-

Tabela 1. Critérios para o início da ISCI de acordo com as diretrizes da Direção Geral de Saúde (DGS)

\begin{tabular}{lcc}
\hline Variável & & \\
\hline HbAlc $>7 \%$ & 36 & $(42,4 \%)$ \\
Complicações microvasculares & 30 & $(35,2 \%)$ \\
Flexibilidade (trabalho e estilo de vida) & 6 & $(7,1 \%)$ \\
Hipoglicemias graves & 5 & $(5,9 \%)$ \\
Variabilidade glicémica & 5 & $(5,9 \%)$ \\
Gravidez & 3 & $(3,5 \%)$ \\
\hline Total & $\mathbf{8 5}$ & \\
\hline
\end{tabular}

Nota: Os dados são apresentados como n (\%). bólico não satisfatório com MIDI (pelo menos 4 injeções diárias de insulina prandial e basal), definido como HbAlc > 7\%, fenómeno de Dawn, níveis de glicose sanguínea $>140-160$ mg/dL (8-9 $\mathrm{mmol} / \mathrm{L}$ ) e elevada variabilidade glicémica; episódios frequentes de hipoglicemia grave ou episódios de hipoglicemia não detetada; necessidade de estilo de vida flexível (p.e. trabalho por turnos, viagens frequentes entre regiões com diferentes fusos horários); gravidez (ou planeamento de gravidez); necessidade de doses baixas de insulina. ${ }^{12-14}$

Os resultados promissores advindos da prática clínica atual, têm vindo a tornar a ISCI no modo terapêutico preferencial, visto melhor mimetizar a ação pancreática fisiológica. Contudo, é limitada a evidência que suporta a sua superioridade relativamente à MIDI. Neste contexto, o objetivo do nosso estudo consistiu em avaliar os efeitos da transição de MIDI para ISCI, no tratamento intensivo da DM tipo 1.

\section{Material e Métodos}

\section{Desenho de estudo}

Estudo observacional longitudinal retrospetivo realizado no Serviço de Endocrinologia do Centro Hospitalar Universitário de São João, em que se avaliou a evolução dos parâmetros clínicos e analíticos dos doentes com DM tipo1 que transitaram de terapêutica intensiva com MIDI para ISCI entre o ano de 2006 e 2014.

Todos os doentes que iniciaram ISCI obedeciam aos critérios da DGS acima descritos, tendo sido submetidos a uma avaliação psicológica e educação terapêutica.

O estudo foi realizado de acordo com os princípios presentes na Declaração de Helsínquia, revista em 2008.

O Comité de Ética desta instituição aprovou o protocolo do estudo, sendo que, os registos dos doentes somente foram acedidos após esta autorização, respeitando os imperativos éticos, deontológicos e de privacidade.

\section{Recolha de dados}

Foram incluídos os diabéticos tipo 1, maiores de 18 anos, que se encontravam há 2 ou mais anos sob terapêutica com ISCI, monitorizados nesta instituição, num total de 85 indivíduos. Estes doentes, previamente ao estudo, encontravam-se em regime de MIDI tendo como alvo terapêutico uma glicose plasmática em jejum e 
pré-prandial compreendida entre $100-120 \mathrm{mg} / \mathrm{dL}$ e $\mathrm{HbA} 1 \mathrm{c}<7,0 \%$.

A informação clínica e analítica foi recolhida, retrospetivamente, para cada doente.

Os eventos de hipoglicemia (glicemia capilar $<70 \mathrm{mg} / \mathrm{dL}$ ) e de hiperglicemia (glicemia capilar pré-prandial $>200 \mathrm{mg} / \mathrm{dL}$ ) foram avaliados antes e após a transição para ISCI, tendo os doentes sido instruídos a realizar 6 pesquisas de glicemia capilar diárias e o respetivo registo efetuado através da descarga de glicómetros referente ao período de 3 meses entre cada consulta. A HbA1c (\%) e a glicose plasmática em jejum $(\mathrm{mg} / \mathrm{dL})$ foram colhidas pré-ISCI, no momento inicial de ISCI (momento 0) e, subsequentemente, aos 6 (momento 6), 12 (momento 12) e 24 (momento 24) meses após o início de ISCI.

$\mathrm{O}$ índice de massa corporal (IMC) $\left(\mathrm{kg} / \mathrm{m}^{2}\right)$ foi calculado com base no peso e estatura dos doentes para os momentos $0,6,12$ e 24 .

Os seguintes parâmetros, tais como, dose diária total (DDT) de insulina, insulina basal e bólus de insulina prandial (unidades de insulina) e fator de sensibilidade à insulina (FSI) (calculada pela regra dos 1800), foram obtidos pré-ISCI, no momento 0,6 , 12 e $24 .{ }^{15} \mathrm{O}$ número de perfis de insulina dos doentes e o rácio de insulina: carbo-hidratos (10 g) foram calculados pré-ISCI e nos momentos $0,6,12$ e 24 , com a regra dos $500 .{ }^{15}$

Os dados referentes às complicações microvasculares, tais como, retinopatia (avaliação oftalmológica), ${ }^{14}$ nefropatia (albuminúria $>30 \mathrm{mg} /$ dia) ${ }^{14}$ e neuropatia (polineuropatia distal simétrica ou alterações detetadas após exame com monofilamento e diapasão), ${ }^{14}$ foram avaliadas como presentes ou ausentes pré-ISCI e nos momentos 0, 6, 12 e 24.

Foram também avaliados os parâmetros relacionados com o perfil lipídico, como colesterol total, LDL-C, HDL-C e triglicerídeos (mg/dL) nos momentos 0, 6, 12 e 24 .

Para a avaliação dos efeitos de ISCI foi ainda efetuada a seguinte análise por subgrupos: HbA1c pré-ISCI $(\leq 7,0 \%$ vs $>$ $7,0 \%$ ); idade ( $\leq 35$ anos $v s>35$ anos); sexo (masculino versus feminino); IMC $\left(\leq 25 v s>25 \mathrm{~kg} / \mathrm{m}^{2}\right)$; duração da doença $(\leq 15$ anos $v s>15$ anos); DDT de insulina $(\leq 45 v s>45$ unidades de insulina); FSI ( $\leq 40 v s>40)$; complicações microvasculares (presença ou ausência).

\section{Análise estatística}

A base de dados e análise estatística foi realizada através do programa Statistical Package for the Social Sciences (SPSS), versão 23.

Para a análise estatística, foram utilizados os testes de Wilcoxon e Mann-Whitney. Os resultados são apresentados como médias \pm desvio padrão, medianas e percentagens. Um valor bilateral de $p<0,05$ foi considerado como estatisticamente significativo.

\section{Resultados}

Foram analisados 85 doentes, 35 do sexo masculino $(41,2 \%)$ e 50 do sexo feminino (58,8\%). A média de idades foi de $38 \pm$ 11 anos (19-77 anos) e a duração média da doença foi de $21 \pm 9$ anos. A caracterização da população em estudo é apresentada na Tabela 2.

Em relação às complicações microvasculares, no início do estudo (momento 0), 29,3\% dos doentes apresentavam retinopatia, $15,9 \%$ nefropatia e $4,9 \%$ neuropatia. Não se observou variação significativa destas complicações ao longo do estudo.

Verificou-se uma diminuição da frequência semanal de hipoglicemias após transição para ISCI $(3,0 \pm 5,0$ vs 2,0 $\pm 2,2, p=$ 0,001 , pré-ISCI versus momento 6), tendo-se também observado
Tabela 2. Características clínicas e demográficas dos doentes

\begin{tabular}{lc}
\hline Variável & 85 \\
\hline $\mathrm{N}$ & $38 \pm 11$ \\
Idade (anos) & $24,53 \pm 3,72$ \\
IMC $\left(\mathrm{kg} / \mathrm{m}^{2}\right)$ (Momento 0)* & $21 \pm 9$ \\
Duração da doença (anos) & $5,0 \pm 2.0$ \\
Período de tempo com ISCI (anos) & \\
Sexo & $35(41,2 \%)$ \\
Masculino & $50(58,8 \%)$ \\
Feminino & \\
Complicações microvasculares (Momento 0) & $4(4,9 \%)$ \\
$\quad$ Neuropatia & $24(29,3 \%)$ \\
$\quad$ Retinopatia & $13(15,9 \%)$ \\
$\quad$ Nefropatia & \\
\hline Os dados são apresentados como média \pm desvio padrão (DP) ou como n (\%). \\
ISCI: infusão subcutânea contínua de insulina; IMC: índice de massa corporal.
\end{tabular}

uma diminuição na frequência semanal de hiperglicemias $(5,5 \pm$ 6,1 vs 2,5 $\pm 2,6, p=0,05$, pré-ISCI versus momento 6).

Constatou-se ainda uma redução significativa dos níveis de HbA1c pré-ISCI quando comparado com o momento $0(0,80 \%, p$ $<0,001)$, contudo não foi observada, globalmente, uma variação significativa dos níveis de HbA1c do momento 0 para os subsequentes momentos do estudo (Tabela 3 e Fig. 1).

Tabela 3. Variação dos valores de HbA1c entre o momento pré-ISCI e os momentos subsequentes.

\begin{tabular}{lcc}
\hline & Variação (\%) & $\begin{array}{c}\text { Teste de } \\
\text { Wilcoxon } \\
\text { (valor de } \boldsymbol{p})\end{array}$ \\
\hline $\begin{array}{l}\text { HbA1c pré-ISCI - HbA1c } \\
\text { Momento 0 (\%) }\end{array}$ & $0,80 \pm 0,21(-1,50-7,60)$ & $<\mathbf{0 , 0 0 1}$ \\
$\begin{array}{l}\text { HbA1c pré-ISCI - HbA1c } \\
\text { Momento 6 (\%) }\end{array}$ & $0,74 \pm 0,18(-1,10-7,60)$ & $<\mathbf{0 , 0 0 1}$ \\
$\begin{array}{l}\text { HbA1c pré-ISCI - HbA1c } \\
\text { Momento 12 (\%) }\end{array}$ & $0,61 \pm 0,27(-2,80-6,30)$ & $<\mathbf{0 , 0 0 1}$ \\
$\begin{array}{l}\text { HbA1c pré-ISCI - HbA1c } \\
\text { Momento 24 (\%) }\end{array}$ & $0,68 \pm 0,22(-1,60-6,50)$ & $<\mathbf{0 , 0 0 1}$ \\
\hline
\end{tabular}

Nota: Os dados são apresentados como média \pm desvio padrão (DP) (min.-máx.) e consistem na diferença entre o valor no momento pré-ISCI e os momentos subsequentes.

ISCI: infusão subcutânea contínua de insulina.

No que respeita à DDT de insulina, FSI, insulina basal e bólus prandial, verificou-se uma redução estatisticamente significativa em todos os momentos do estudo por comparação ao pré-ISCI ( $p$ $=0,001$ ), exceto no bólus prandial 12 meses após ISCI (Fig. 2). Não se observaram diferenças estatisticamente significativas relativamente ao rácio de insulina: carbo-hidratos e perfis de insulina.

Quanto ao perfil lipídico, verificou-se um aumento significativo dos níveis de HDL $(+2,81 \pm 10,34 \mathrm{mg} / \mathrm{dL})$ no momento $12 \mathrm{em}$ relação ao momento $0(60,62 \pm 14,99$ vs $57,85 \pm 11,57 \mathrm{mg} / \mathrm{dL}, p$ $=0,039$, respetivamente). Não foram reportadas diferenças significativas para os outros momentos de ISCI quanto ao HDL, nem para os outros parâmetros do perfil lipídico em todos os momentos de estudo (colesterol total, LDL, triglicerídeos) (Fig. 3).

Relativamente ao IMC, não foi observada variação estatistica- 


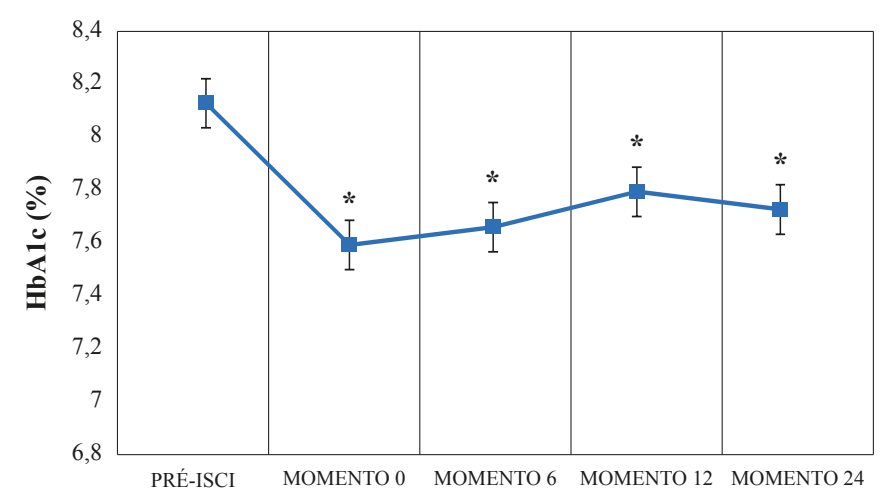

Figura 1. HbA1c (\%) em valores de mediana ao longo dos momentos de estudo. * $p<0,001$ em comparação com o valor do regime de MIDI (Pré-ISCI).

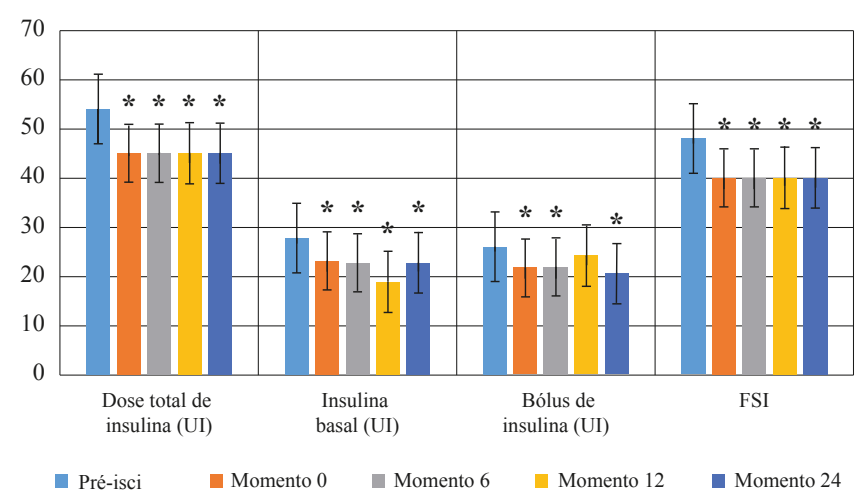

Figura 2. Variação dos valores de mediana dos parâmetros de ISCI ao longo dos momentos de estudo. FSI: fator de sensibilidade à insulina.

* $p<0,001$ em comparação com o valor do regime de MIDI (Pré-ISCI).

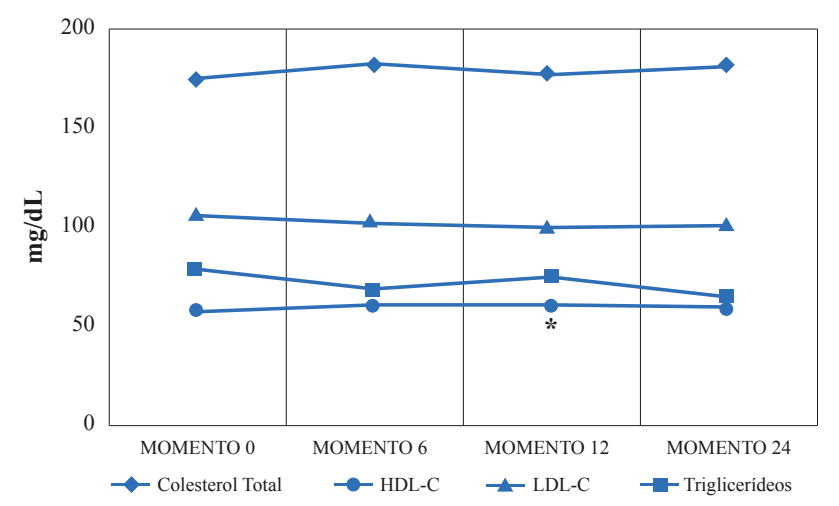

Figura 3. Variação dos valores de mediana do perfil lipídico ao longo dos momentos de estudo.

$* p=0,039$ em comparação com o momento 0 .

mente significativa, ao longo do período de estudo.

Os níveis de creatinina apresentaram um aumento significativo de $0,05 \pm 0,16 \mathrm{mg} / \mathrm{dL}$ no momento 6 em relação ao momento $0(0,96 \pm 0,35 v s 0,91 \pm 0,32 \mathrm{mg} / \mathrm{dL}, p=0,05)$. Por outro lado, verificou-se uma diminuição significativa de $0,05 \pm 0,28 \mathrm{mg} / \mathrm{dL}$ no momento $12(0,86 \pm 0,37$ vs $0,91 \pm 0,32 \mathrm{mg} / \mathrm{dL}, p=0,015)$ e de $0,14 \pm 0,15 \mathrm{mg} / \mathrm{dL}$ no momento $24(0,77 \pm 0,37$ vs $0,91 \pm 0,32 \mathrm{mg} /$ $\mathrm{dL}, p=0,015$ ), relativamente ao momento 0 . Não foi observada variação significativa dos níveis de albuminúria durante o período do estudo.

No que respeita à análise por subgrupos, os testes de Mann-
-Whitney não revelaram diferenças estaticamente significativas para a idade, complicações microvasculares e DDT de insulina durante os 24 meses de follow-up.

O subgrupo de doentes com HbAlc $>7 \%$ obteve um maior benefício metabólico relativamente ao grupo de doentes com $\mathrm{HbAlc}$ $\leq 7 \%(\Delta \mathrm{HbA} 1 \mathrm{c}=-0,55 \%$ vs $0,20 \%$, respetivamente, $p<0,05)$, nos 6 primeiros meses após ISCI. Constatou-se ainda que, neste período, o subgrupo com melhor controlo metabólico, apresentou uma melhoria no perfil lipídico comparativamente com o subgrupo de pior controlo metabólico. Com efeito, verificou-se um maior aumento dos níveis de HDL ( $4 \mathrm{mg} / \mathrm{dL} v s 1 \mathrm{mg} / \mathrm{dL} ; p=0,013)$ e uma diminuição superior dos níveis de LDL $(-8 \mathrm{mg} / \mathrm{dL} v s-2 \mathrm{mg} / \mathrm{dL}$, $p=0,044)$, para os doentes melhor controlados. Nos momentos posteriores do estudo não foram reportadas variações, estatisticamente significativas, nos níveis de HbA1c e perfil lipídico.

No subgrupo de doentes com FSI $>40$, contrariamente ao subgrupo de FSI $\leq 40$, verificou-se uma diminuição significativa dos níveis de triglicerídeos entre os momentos 0 e 24 (-10 mg/dL vs 9 $\mathrm{mg} / \mathrm{dL}, p=0,008)$.

No que respeita ao subgrupo de doentes com tempo de doença $\leq 15$ anos, observou-se, entre os momentos 0 e 12 , um maior aumento dos níveis de triglicerídeos, relativamente ao subgrupo com > 15 anos $(10 \mathrm{mg} / \mathrm{dL} v s 4 \mathrm{mg} / \mathrm{dL}, p=0,026)$, não se tendo verificado variações estatisticamente significativas nos momentos subsequentes do estudo.

Em relação ao subgrupo de indivíduos com IMC $\leq 25 \mathrm{~kg} / \mathrm{m}^{2}$, a variação entre o momento 0 e 24 revelou uma diminuição dos níveis de triglicerídeos contrariamente aos indivíduos com IMC > $25 \mathrm{~kg} / \mathrm{m}^{2}(-10 \mathrm{mg} / \mathrm{dL}$ vs $5 \mathrm{mg} / \mathrm{dL}, p=0,033)$.

Não foram reportados outros resultados estatisticamente significativos para as restantes variáveis avaliadas neste estudo.

Não se observaram episódios de coma hipoglicémico, nem de cetoacidose diabética, durante todo o período de estudo.

\section{Discussão}

Analisando os resultados do nosso estudo, verificou-se que, a terapêutica com ISCI demonstrou ser mais eficaz comparativamente à MIDI, nos doentes com pior controlo metabólico, traduzido pela redução estatisticamente significativa em $0,55 \%$ dos níveis de $\mathrm{HbAlc}$, nos primeiros 6 meses de ISCI. Observou-se uma estabilização, deste parâmetro, nos momentos posteriores do estudo. Por outro lado, na análise global, não se observou uma variação significativa nos níveis de HbA1c após o momento 0 . Deste modo, estes resultados indicam que a terapêutica com ISCI é a melhor escolha no tratamento da DM tipo $1 \mathrm{em}$ doentes com inadequado controlo metabólico. Estes dados estão de acordo com outros estudos anteriormente publicados. ${ }^{16}$

É sabido que esta terapêutica, ao permitir uma considerável redução da DDT de insulina (de $15 \%$ a $20 \%$ ), libertada de forma constante, com uma programação personalizada e ajustada às necessidades individuais, proporciona uma maior flexibilidade e qualidade de vida ao doente, mimetizando de forma mais eficaz a secreção fisiológica de insulina. ${ }^{17}$ Consequentemente, era já expectável uma significativa redução na frequência semanal dos episódios de hipo e de hiperglicemia, juntamente com a ausência de hiperglicemia grave e cetoacidose durante a duração do estudo.

De salientar que os doentes elegíveis para ISCI (ou familiares em caso de crianças) necessitam ser capazes de efetuar corretamente contagem de carbo-hidratos e cálculo da dose a administrar, sendo autónomos no manuseamento da bomba infusora de insulina. Neste estudo, o facto de cada doente ter sido avaliado ao 
longo dos diferentes momentos estabelecidos, permitiu minimizar as diferenças interindividuais associadas à motivação, dieta, peso e atividade física.

No presente estudo, a redução da DDT de insulina, basal e bólus prandial, constituiu uma das vantagens da terapêutica com ISCI e manteve-se durante todo o período de estudo, o que está de acordo com a evidência existente na literatura. ${ }^{17,18}$

No que respeita ao perfil lipídico, os resultados obtidos demonstraram um efeito favorável, com aumento significativo do HDL a nível global (momento 12) e no subgrupo com melhor controlo metabólico (momento 6). Para além disso, foi encontrado um decréscimo significativo nos níveis de LDL para o mesmo subgrupo de doentes. Adicionalmente, verificou-se uma diminuição considerável dos níveis de triglicerídeos no subgrupo de doentes com FSI $>40$, ao longo de todo o estudo. Na verdade, o facto destes doentes apresentarem uma menor resistência à insulina poderá estar relacionado com a melhoria do controlo metabólico e do perfil lipídico.

No que concerne ao IMC, não se verificou variação significativa com a terapêutica de ISCI durante o estudo, em conformidade com dados anteriormente publicados. ${ }^{13}$ No subgrupo de doentes com IMC $<25 \mathrm{~kg} / \mathrm{m}^{2}$ observou-se uma diminuição dos triglicerídeos, comparativamente com os indivíduos com excesso de peso. Estes resultados são consistentes com a alteração do perfil lipídico associado aos fenómenos inerentes à obesidade. ${ }^{19}$

Atualmente a aposta na terapêutica com ISCI é reconhecida por possuir várias vantagens, sendo mais económica a longo prazo no tratamento da DM tipo 1. Estes dados são sustentados por vários estudos que sublinham a superioridade da terapêutica com ISCI na DM tipo $1 .^{20-22}$

À parte do benefício evidente sobre o controlo metabólico, a ISCI permite também uma melhor qualidade de vida do doente, tal como já demonstrado em vários estudos. ${ }^{14,20-22}$ Com efeito, mais que uma alternativa à MIDI, esta modalidade terapêutica é hoje considerada como primeira linha no tratamento da DM tipo $1 .^{20-22}$

Dada a crescente universalização desta terapêutica na prática clínica, torna-se imperiosa a consciencialização das possíveis complicações associadas à ISCI, efetuando um seguimento apertado do doente a fim de identificar possíveis falhas no tratamento. Neste sentido, com as intervenções apropriadas, poderemos alcançar os objetivos desejáveis através de programas de educação terapêutica contínua, fundamentais para o sucesso da terapêutica com ISCI. ${ }^{23}$

Não obstante a maioria dos doentes atingir resultados satisfatórios com ISCI, alguns poderão não responder positivamente à mesma, tornando a ISCI, para estes, uma alternativa inadequada. Este facto levanta a questão de existirem potenciais preditores de deterioração ou ausência de efeitos favoráveis com ISCI. ${ }^{23}$ Juntamente com os fatores intrínsecos ao doente, também as restrições económicas constituem uma importante limitação para a implementação generalizada deste tipo de terapêutica, ainda que, como acima referido, a mesma demonstre economizar recursos a longo prazo. $^{20}$

\section{Conclusão}

Dadas as crescentes evidências da eficácia da ISCI no controlo metabólico e resultados promissores nas análises de custo-benefício existentes na literatura, esta terapêutica é hoje reconhecida como uma estratégia de primeira linha, no tratamento intensivo da DM tipo 1. Os resultados do nosso estudo estão em concordância com dados anteriormente publicados que enfatizam ser mais acentuado este benefício nos doentes com inadequado controlo glicémico e/ ou episódios frequentes de hipo e hiperglicemia. Este facto poderá dever-se a uma melhor cinética da insulina, com menor variabilidade na absorção e maior reprodutibilidade dos níveis glicémicos, nomeadamente em comparação com o tratamento por MIDI.

Não obstante os resultados animadores associados à ISCI, não é ainda claro todo o potencial impacto deste tipo de terapêutica, a longo prazo, no que respeita ao risco cardiovascular e outras possíveis lesões em órgãos-alvo. Torna-se, assim, imperativo a realização futura de estudos longitudinais mais alargados por forma a permitir a avaliação da real eficácia relativa da ISCI, no tratamento da DM tipo 1.

\section{Responsabilidades Éticas}

Conflitos de Interesse: Os autores declararam a inexistência de conflitos de interesse

Fontes de Financiamento: Não existiram fontes externas de financiamento para a realização deste artigo.

Confidencialidade dos Dados: Os autores declaram ter seguido os protocolos do seu centro de trabalho acerca da publicação dos dados de doentes.

Proteção de Pessoas e Animais: Os autores declaram que os procedimentos seguidos estavam de acordo com os regulamentos estabelecidos pelos responsáveis da Comissão de Investigação Clínica e Ética e de acordo com a Declaração de Helsínquia da Associação Médica Mundial.

Proveniência e Revisão por Pares: Não comissionado; revisão externa por pares.

\section{Ethical Disclosures}

Conflicts of Interest: The authors report no conflict of interest. Funding Sources: No subsidies or grants contributed to this work. Confidentiality of Data: The authors declare that they have followed the protocols of their work center on the publication of patient data

Protection of Human and Animal Subjects: The authors declare that the procedures followed were in accordance with the regulations of the relevant clinical research ethics committee and with those of the Code of Ethics of the World Medical Association (Declaration of Helsinki).

Provenance and Peer Review: Not commissioned; externally peer reviewed.

\section{Referências}

1. American Diabetes Association. Diagnosis and classification of diabetes mellitus. Diabetes Care. 2014; 37:S81-90.

2. You WP, Henneberg M. Type 1 diabetes prevalence increasing globally and regionally: the role of natural selection and life expectancy at birth. BMJ Opn Diabetes Res Care. 2016;4:e000161. doi: 10.1136/ bmjdrc-2015-000161.

3. I International Diabetes Federation. Diabetes Atlas. Brussels: IDF; 2015.

4. Zhang P, Zhang X, Brown J. Global healthcare expenditure on diabetes for 2010 and 2030. Diabetes Res Clin Pract. 2010; 87: 293-301. doi: 10.1016/j.diabres.2010.01.026.

5. American Diabetes Association. Standards of medical care in diabetes 2019. Diabetes Care. 2019; 42(Suppl 1): S1-S2. doi: 10.2337/dc19-S016.

6. Aronson D. Hyperglycemia and the pathobiology of diabetic complications. Adv Cardiol. 2008; 45: 1-16.

7. Esteves C, Neves C, Carvalho D. A hipoglicemia no diabético: controvérsia na avaliação, à procura das suas implicações. Acta Med Port. 2012; 25: 454-60. 
8. Danne T, Bolinder J. New insulins and insulin therapy. Diabetes Technol Ther. 2014; 16 : S34-43. doi: 10.1089/dia.2014.1505.

9. Garg SK, Shah VN. Newer therapies for diabetes management. Diabetes Technol Ther. 2014; 16: S119-127. doi: 10.1089/dia.2014.1514.

10. Tumminia A, Crimi S, Sciacca L, Buscema M, Frittitta L, Squatrito S, et al.Efficacy of real-time continuous glucose monitoring on glycaemic control and glucose variability in Type 1 diabetic patients treated with either insulin pumps or multiple insulin injection therapy: a randomised controlled cross-over trial. Diabetes Metab Res Rev. 2015; 31: 61-8. doi: 10.1002/dmrr.2557.

11. Atlas E, Thorne A, Lu K. Closing the loop. Diabetes Technol Ther. 2014; 16: S23-33. doi: 10.1089/dia.2014.1504.

12. Direção Geral de Saúde. Circular Normativa $n^{\circ}$ 17/DSCS/DGID. Gestão Integrada da Diabetes - Elegibilidade dos doentes para tratamento através de perfusão subcutânea contínua de insulina. Lisboa: DGS; 2008.

13. Pickup JC, Yemane N, Brackenridge A, Pender S. Nonmetabolic complications of continuous subcutaneous insulin infusion: a patient survey. Diabetes Technol Ther. 2014; 16: 145-9. doi: 10.1089/ dia.2013.0192

14. Balsa AM, Neves C, Alves M. Continuous subcutaneous insulin infusion. Acta Med Port. 2011; 24: 147-56.

15. Simonson DC, Tamborlane WV, Sherwin RS, Smith JD, DeFronzo RA. Improved insulin sensitivity in patients with type I diabetes mellitus after CSII. Diabetes. 1985; 34: 80-6.

16. Umpierrez GE, Klonoff DC. Diabetes technology update: use of insulin pumps and continuous glucose monitoring in the hospital. Diabetes Care.
2018; 41: 1579-89. doi: 10.2337/dci18-0002.

17. Ross PL, Milburn J, Reith DM. Clinical review: insulin pump-associated adverse events in adults and children. Acta Diabetol. 2015; 52: 1017-24. doi: 10.1007/s00592-015-0784-2.

18. Walsh J, Roberts R, Bailey T. Guidelines for insulin dosing in continuous subcutaneous insulin infusion using new formulas from a retrospective study of individuals with optimal glucose levels. J Diabetes Sci Technol. 2010; 4: 1174-81.

19. Ladeia AM, Adan L, Couto-Silva AC, Hiltner A, Guimarães AC. Lipid profile correlates with glycemic control in young patients with type 1 diabetes mellitus. Prev Cardiol.2006; 9:82-8.

20. Roze S, Smith-Palmer J, Valentine W, de Portu S, Nørgaard K, Pickup JC. Cost-effectiveness of continuous subcutaneous insulin infusion versus multiple daily injections of insulin in Type 1 diabetes: a systematic review. Diabet Med. 2015; 32: 1415-24. doi: 10.1111/dme.12792.

21. Cohen ND, Hong ES, Van Drie C, Balkau B, Shaw J. Long-term metabolic effects of continuous subcutaneous insulin infusion therapy in type 1 diabetes. Diabetes Technol Ther 2013; 15: 544-49. doi: 10.1089/ dia.2012.0331

22. Anyanwagu U, Olaoye H, Jennings P, Ashton-Cleary S, Sugunendran S, Hughes D,et al. Continuous subcutaneous insulin infusion (CSII) therapy at Derby Teaching Hospitals: sustained benefits in glucose control. Diabet Med. 2017; 34: 1154-7. doi: 10.1111/dme.13367.

23. Nixon R, Folwell R, Pickup JC. Variations in the quality and sustainability of long-term glycaemic control with continuous subcutaneous insulin infusion. Diabet Med. 2014; 31:1174-7. doi: 10.1111/dme.12486. 